\title{
Diversité des logiques de fonctionnement et durabilité des exploitations en agriculture biologique
}

Diversity of operating logics and sustainability of organic farms

\section{Mohamed Gafsi et Jean-Luc Favreau}

\section{(2) OpenEdition}

Journals

Édition électronique

URL : http://journals.openedition.org/economierurale/4269

DOI : $10.4000 /$ economierurale.4269

ISSN : 2105-2581

Éditeur

Société Française d'Économie Rurale (SFER)

Édition imprimée

Date de publication : 20 janvier 2014

Pagination : 129-143

ISSN : 0013-0559

\section{Référence électronique}

Mohamed Gafsi et Jean-Luc Favreau, « Diversité des logiques de fonctionnement et durabilité des exploitations en agriculture biologique », Économie rurale [En ligne], 339-340 I janvier-mars 2014, mis en ligne le 20 janvier 2016, consulté le 19 avril 2019. URL : http://journals.openedition.org/ economierurale/4269; DOI : 10.4000/economierurale.4269 


\title{
Diversité des logiques de fonctionnement et durabilité des exploitations en agriculture biologique
}

\author{
Mohamed GAFSI, Jean-Luc FAVREAU • UMR Dynamiques Rurales, École Nationale de Formation \\ Agronomique, Castanet-Tolosan \\ mohamed.gafsi@educagri.fr ; jean-luc.favreau@educagri.fr
}

Les conceptions de l'agriculture biologique et les modèles de son développement présentent une grande diversité. L'objectif de cet article est de saisir cette diversité au niveau des exploitations agricoles à travers la notion de "logique de fonctionnement ", et de montrer par la suite que la durabilité de ces exploitations varie en fonction des logiques poursuivies. Une typologie a été réalisée à partir d'une méthode d'analyse en composantes principales. Elle a permis d'identifier trois grandes logiques de fonctionnement : entrepreneuriale, recherche d'équilibre et modernisation. Ces logiques présentent des différences significatives de durabilité au niveau agro-écologique et économique. Les exploitations poursuivant la logique médiane, de recherche d'équilibre, semblent réussir par différents arbitrages à concilier des objectifs divers et aboutir à une meilleure durabilité.

MOTS-CLÉS : exploitation en agriculture biologique, durabilité, logique de fonctionnement, diversité, typologie

\section{Diversity of operating logics and sustainability of organic farms}

Organic sector has a wide variety of models of development. The purpose of this paper is to capture this diversity at the farm's level, using the notion of operating logic, and to show that the sustainability of these farms depends on the operating logic followed. Three major operating logics are identified from a principal components analysis method: entrepreneurial, compromise and modernization. Farms adopting these logics have significant differences in their sustainability marks, particularly at the agro-ecological and economic dimensions. Farms pursuing the median logic -research a balance- seem to succeed to reconcile different objectives and improve their sustainability. (JEL: Q12, L21, M10).

KEYWORDS: Organic farm, sustainability, operating logic, diversity, typology

D epuis la fin des années 1990, l'agriculture biologique (AB) a connu un important développement en France. Les données de l'Agence Bio (2012) montrent que les principaux indicateurs ont plus que doublé entre 2001 et 2011. Ainsi, le nombre d'exploitations $\mathrm{AB}$ est passé de 10364 à $23135(+123 \%)$, la surface agricole utile (SAU) de 419750 à 975141 ha (+ 132\%) et le nombre d'opérateurs d'aval (transformateurs et distributeurs) de 5100 à 12131 (+138\%). Ce développement de l'AB est soutenu à la fois par la demande croissante et continue de produits biologiques et par l'accompagnement des politiques publiques. Le marché des produits biologiques a quadruplé en dix ans pour atteindre quatre milliards d'euros en 2011, soit un taux de croissance annuel d'environ $11 \%$ (Agence Bio, 2012). Pour les politiques publiques, le ministère de l'Agriculture a mis en place trois principaux plans de développement de l'AB (le « Plan Pluriannuel de Développement de l'AB 1998-2006 » en 1998, le plan «Agriculture biologique : horizon 2012 » en 2009 et le plan « Ambition bio 2017 » en 2013).

Mais ce développement de l'AB mérite d'être relativisé et questionné, et ceci pour deux raisons principales. Premièrement, 
même si le développement récent de l'AB est important, il demeure, tout de même, en deçà des objectifs ambitieux fixés par les plans successifs des pouvoirs publics ${ }^{1}$. L'AB ne représente en 2011 que 4,5\% des exploitations agricoles en France et 3,5\% de la SAU globale. Le marché des produits biologiques représente en 2011 environ $2,6 \%$ de la consommation alimentaire en France (Agence Bio, 2012). Les différentes filières de l'AB (céréales, légumes, lait, viande) se structurent lentement et non sans difficultés (Schieb-Bienfait et Sylvander, 2004 ; Bréchet et Schieb-Bienfait, 2006). Les producteurs rencontrent différents types de difficultés (techniques, économiques, financières) pour répondre à la demande croissante. Ainsi, malgré le contexte de développement rapide de l'AB, des questions relatives à la durabilité de ce mode de production restent posées (Rigby et Caceres, 2001 ; Gafsi et al., 2010 ; Goldberger, 2011).

Deuxièmement, le récent développement de l'AB a fait émerger de nouveaux enjeux en lien avec la gouvernance et le risque de mutation identitaire de ce secteur (Pernin, 1998). En effet, ce fort développement a rendu l'AB très attractive, ces dernières années, pour beaucoup d'acteurs agricoles et agroalimentaires (Leroux, 2011). Cette évolution suscite chez des acteurs historiques de l'AB des craintes d' « industrialisation » de l'AB, ce qui est formulé dans le milieu scientifique par la thèse de la « conventionalisation » (Guthman, 2004 ; Kratochvil et Leitner, 2005 ; Lockie et Halpin, 2005 ; Darnhofer et al., 2010a). De ce fait, l'AB en France n'est pas homogène ; on peut difficilement parler de l'AB, mais plutôt des agricultures biologiques. Loin de l'opposition classique entre une $\mathrm{AB}$ 《éthique » et une

1. Par exemple le Plan Barnier de 2009, qui prévoyait le triplement de la surface en AB en 2012, pour atteindre $6 \%$ de la SAU, et le développement de l'AB dans la restauration collective publique pour atteindre $20 \%$ d'approvisionnement. autre « opportuniste », plusieurs conceptions de l'AB, et par conséquent une pluralité de modèles de développement coexistent (Sylvander et al., 2006 ; Lamine et Bellon, 2009). La durabilité des exploitations biologiques est-elle la même indépendamment du modèle qu'elles adoptent ?

L'objectif de ce papier est double : mettre en évidence dans un premier temps la diversité des logiques de fonctionnement des exploitations biologiques, et montrer dans un deuxième temps que la durabilité des exploitations varie en fonction des types de logiques de fonctionnement. Nous partons de l'idée que ces logiques traduisent l'inscription des exploitants dans les différentes conceptions de l'AB. Les matériaux empiriques de cette recherche sont constitués d'enquêtes réalisées auprès d'exploitations biologiques de différentes orientations technico-économiques de Midi-Pyrénées en 2011 et 2012. Nous avons privilégié une approche méthodologique quantitative pour analyser la diversité des logiques de fonctionnement des exploitations et apprécier leur durabilité.

Après avoir présenté le cadre théorique, qui inscrit notre approche dans le courant de la gestion centrée sur les pratiques des acteurs et leurs projets (Brossier et al., 1997), nous décrivons le terrain d'étude et la méthodologie. Nous exposerons ensuite les résultats concernant la diversité des logiques de fonctionnement et l'appréciation de la durabilité, avant d'apporter des éléments de discussion et de conclusion.

\section{Diversité et durabilité de l'agriculture biologique}

\section{Diversité de conceptions de I'AB et logiques de fonctionnement}

Bien que pratiquée depuis les années 1960, l'AB n'a été reconnue officiellement en France qu'en début des années 1980, avec la loi d'orientation agricole qui la définit comme « une agriculture qui n'utilise pas de produits chimiques de synthèse ». Cette définition 
réglementaire et normative est complétée par une approche plus globale, défendue par les acteurs historiques de l'AB dont la Fédération nationale de l'AB (FNAB), qui insiste sur le respect du vivant et des cycles naturels, en valorisant les équilibres écosystémiques et les conditions agro-écologiques locales. Cette approche globale considère aussi que l'AB est un mouvement social (Van Dam, 2005 ; Leroux, 2011) qui vise à promouvoir des relations justes et une bonne qualité de vie pour tous. L'IFOAM ${ }^{2}$, quant à elle, énonce quatre principes : la santé, l'écologie, l'équité et la précaution.

\section{Diversité de conceptions et de modèles}

L'AB est traversée par plusieurs tensions et dichotomies (Streith et al., 2011). La plus célèbre, ces dernières années, est l'opposition $\mathrm{AB}$ 《éthique » / $\mathrm{AB}$ « économique ». La première serait militante, pratiquant les principes biologiques par conviction et depuis longtemps ; la seconde serait plus opportuniste, reconvertie récemment à l' $\mathrm{AB}$ pour des raisons économiques. Certes, cette opposition de conceptions existe à des degrés variables et se traduit par les modèles de substitution d'intrants et de reconception de système (Lamine et Bellon, 2009). Toutefois, il est insuffisant de réduire la diversité de conceptions à cette distinction binaire. Plusieurs travaux empiriques en France comme en Europe, ont montré une diversité croissante de conceptions et donc de modèles de développement de l'AB (Morel et Le Guen, 2003 ; Van Dam, 2005 ; Ruault, 2006 ; Sylvander et al., 2006 ; Darnhofer et al., 2010a). Sylvander et al. (2006) distinguent quatre modèles de développement. Morel et Le Guen (2003) ont proposé une typologie de cinq types de producteurs correspondant à des modèles

2. International Federation of Organic Agriculture Movements; « Nature \& progrès » est à l'origine de la création de l'IFOAM en 1972 ; http:// www.ifoam.org/about_ifoam/principles/index. html (consulté en avril 2013). différents de l'AB : entrepreneurs, opportunistes, contestataires, producteurs en relance professionnelle, repreneurs professionnels.

\section{Logique de fonctionnement pour saisir la diversité}

L'inscription dans un modèle de l'AB se traduit concrètement, pour un agriculteur, par les choix stratégiques concernant le développement de son exploitation et par ses pratiques agronomiques et économiques dans la conduite de cette exploitation. Pour saisir la diversité des modèles et des conceptions de l'AB, nous mobilisons la notion de logique de fonctionnement comme cadre d'analyse. Cette notion fait référence au concept de fonctionnement de l'exploitation agricole. Elle peut être définie comme étant « les raisons qui fondent la cohérence globale des pratiques de l'agriculteur dans la conduite de son exploitation agricole ». $\mathrm{Ce}$ choix méthodologique s'inscrit globalement dans l'analyse systémique de l'exploitation agricole et plus particulièrement dans l'approche de la gestion centrée sur les pratiques des acteurs et leurs projets (Brossier et al., 1997). Le concept de « fonctionnement de l'exploitation agricole » est central dans cette approche. Capillon (1988,) le définit comme suit : " enchaînement de prises de décisions de la part de l'agriculteur en vue d'atteindre un ou plusieurs objectifs qui régissent des processus de production dans un ensemble de contraintes ». Ce concept a fondé la méthode de typologie de fonctionnement élaborée par des économistes (Brossier et Petit, 1977) et des agronomes (Capillon, 1993). La logique de fonctionnement est identifiée à partir de l'analyse des pratiques agricoles qui sont le résultat à la fois du projet de l'agriculteur et de la situation de l'exploitation. Précisons enfin que la notion de logique de fonctionnement est proche de celle de logique d'action, qui cherche à saisir les raisons d'agir de l'acteur (Amblard $e t$ al., 2005). Sauf que la logique de fonctionnement privilégie une approche managériale en ciblant l'exploitation agricole en tant 
qu'unité d'analyse, plutôt que l'agriculteur en tant qu' acteur individuel.

\section{2. $A B$ et durabilité}

Bien que ses définitions soient multiples, il est établi maintenant qu'en agriculture la durabilité est une notion complexe et globale qui comprend simultanément trois dimensions : économique, écologique et sociale. Nous retenons la définition suivante de l'agriculture durable : la capacité du système agricole à maintenir sa productivité et son utilité pour la société dans le long terme. Cette définition comprend deux volets : la durabilité autocentrée, qui vise la pérennité à long terme du système agricole lui-même (Hansen et Jones, 1996), et la durabilité étendue qui concerne la contribution de ce système à la durabilité du territoire local auquel il appartient (Godard et Hubert, 2002).

- Dans le domaine de l'AB, beaucoup de travaux de recherche se sont centrés sur les aspects agronomiques et environnementaux (Tuomisto et al., 2012), sans poser pleinement la question des modèles de développement au sein de l'AB. L'intérêt d'une réflexion en termes de durabilité globale, intégrant les trois dimensions classiques (écologique, économique et sociale) évoquées ci-dessus, est de nous obliger à comprendre par quels arbitrages et quelles combinaisons les agriculteurs en $\mathrm{AB}$ articulent des objectifs et des pratiques parfois contradictoires.

- L'approche globale retenue pour l'appréciation de la durabilité de l'AB fait également écho aux notions d' « intégrité fonctionnelle » (Thompson, 1997) et de " système complexe adaptatif » (Darnhofer et al., 2010b). Dans cette perspective, on ne s'attache pas seulement à regarder les performances techniques, économiques ou même sociales, mais on met en évidence les pratiques agricoles qui valorisent les interactions entre les écosystèmes au niveau de l'exploitation agricole. Cet aspect est très important dans le cas de l'AB, comme nous l'avons vu dans sa définition plus large. Ainsi, l'aspect agronomique occupe une attention particulière dans l'appréciation de la durabilité des exploitations en $\mathrm{AB}$.

\section{Terrain et méthodologie}

\section{Une $A B$ régionale diversifiée}

En 2011, les huit départements de la Région Midi-Pyrénées comptaient 118700 ha en $\mathrm{AB}(5,2 \%$ de la SAU totale) dont le cinquième en conversion, et 2430 exploitations (Agence Bio, 2012). Les productions AB les plus représentées sont les grandes cultures (essentiellement céréales et oléagineux), l'élevage de bovins viande, et à un moindre degré les ovins viande, la production laitière bovine et ovine, le maraîchage. Au-delà de cette diversité de production, l'AB de Midi-Pyrénées est aussi porteuse d'une diversité de situations et de caractéristiques (Leroux, 2011) : diversité du profil sociologique des agriculteurs et de leur ancienneté dans l'AB, diversité des productions, des conditions pédo-climatiques et donc des modes d'organisation spatiale des exploitations, diversité d'organisation des filières et des modes d'organisation et d'encadrement des producteurs. On peut faire l'hypothèse que cette pluralité de situations et de caractéristiques va dans le sens d'une différenciation des logiques de fonctionnement des exploitations. L'étude de terrain a porté sur 74 exploitations en $\mathrm{AB}$ de la région Midi-Pyrénées.

\section{Méthodologie}

Les 74 exploitations ont été choisies de manière aléatoire et sur la base de la coopération des agriculteurs, tout en veillant à respecter la diversité de production de l'AB dans la région. Le tableau 1 présente les principales caractéristiques des exploitations étudiées. Sans prétendre à la représentativité de l'AB régionale, toutes les productions sont 
RECHERCHES

Mohamed GAFSI, Jean-Luc FAVREAU

présentes ; les exploitations ne sont globalement pas de grandes dimensions en termes de SAU ; sauf pour les grandes cultures, beaucoup d'exploitations pratiquent la commercialisation en circuits courts.

Les données ont été collectées par enquête auprès des agriculteurs en 2010 et
2011. L'enquête comprend plusieurs volets couvrant les données nécessaires pour caractériser l'exploitation, saisir sa logique de fonctionnement et apprécier sa durabilité.

L'étude des logiques de fonctionnement des exploitations a été faite à partir de l'analyse des pratiques des agriculteurs

Tableau 1. Caractéristiques des exploitations AB étudiées

\begin{tabular}{lcccccc}
\hline & $\begin{array}{c}\text { Grandes } \\
\text { cultures }\end{array}$ & Maraîchage & Bovins & Ovins & Chèvres & Total \\
\hline Nombre d'exploitations & 23 & 8 & 21 & 14 & 8 & 74 \\
SAU moyenne (ha)* & 93 & 6 & 71 & 154 & 50 & 84 \\
Âge moyen du chef d'exploitation & 47 & 46 & 50 & 49 & 49 & 49 \\
UTH moyen* & 1,3 & 1,7 & 1,7 & 1,7 & 2 & 1,6 \\
CA moyen (milliers d' $€$ )* & 108 & 49 & 121 & 97 & 68 & 99 \\
EBE moyen (milliers d' $€$ * & 44 & 24 & 46 & 37 & 28 & 39 \\
Part moyenne des ventes en circuits & 14 & 95 & 59 & 31 & 94 & 47 \\
courts dans le CA (\%) & & & & & & \\
\hline
\end{tabular}

Note : * SAU : surface agricole utile ; UTH : unité de travail humain, CA : chiffre d'affaires ; EBE : excédent brut d'exploitation

Source : les auteurs.

Tableau 2. Variables pour l'étude des logiques de fonctionnement

\begin{tabular}{|c|c|c|}
\hline Pratique & Caractérisation & Critère de mesure \\
\hline \multicolumn{3}{|l|}{ Agronomique } \\
\hline Intensification & $\begin{array}{l}\text { Degré d'utilisation de l'azote orga- } \\
\text { nique }\end{array}$ & Quantité d'azote / ha fertilisé \\
\hline Autonomie & $\begin{array}{l}\text { Autonomie par rapport aux intrants } \\
\text { (engrais ou aliments pour animaux) }\end{array}$ & $\begin{array}{l}\text { Montant des achats d'intrants / CA } \\
\text { global* }\end{array}$ \\
\hline $\begin{array}{l}\text { Fertilité } \\
\text { agrobiologique }\end{array}$ & $\begin{array}{l}\text { Fertilisation par la combinaison de } \\
\text { trois pratiques: } \\
\text { - Valorisation de la matière orga- } \\
\text { nique (fumier, engrais vert, paille) } \\
\text { - Longueur des rotations } \\
\text { - Part des légumineuses dans } \\
\text { l'assolement }\end{array}$ & $\begin{array}{l}\text { Somme des notes obtenues pour : } \\
\text { - } \% \text { SAU recevant la matière organique } \\
\text { (Note : } 0 \text { pour }<10 \%, 5 \text { pour } 10 \%-20 \% \text {, } \\
8 \text { pour } 20 \%-30 \%, 11 \text { pour }>30 \% \text { ) } \\
\text { - Nombre d'espèces dans la rotation } \\
\text { (Note : } 2 \text { x nombre d'espèces) } \\
\text { - } \% \text { de légumineuses / SAU } \\
\text { (Note : } 0 \text { pour }<10 \%, 2 \text { pour } 10 \%-20 \% \text {, } \\
4 \text { pour } 20 \%-30 \%, 6 \text { pour }>30 \% \text { ) }\end{array}$ \\
\hline \multicolumn{3}{|l|}{ Économique } \\
\hline Capitalisation & $\begin{array}{l}\text { Degré de modernisation et d'inves- } \\
\text { tissement dans l'outil de production } \\
\text { (équipements, bâtiments, installations) }\end{array}$ & Capital d'exploitation / UTH** \\
\hline Spécialisation & $\begin{array}{l}\text { Degré de spécialisation en termes de } \\
\text { production }\end{array}$ & $\%$ Production principale / CA global \\
\hline Commercialisation & $\begin{array}{l}\text { Pratique de commercialisation en } \\
\text { circuit court }\end{array}$ & $\%$ CA en circuit court / CA global \\
\hline
\end{tabular}

Note : * CA global sans primes, ** le capital d'exploitation est le montant de l'actif de l'exploitation sans la valeur du foncier en propriété 
(Capillon, 1988 ; Girard, 2006). Ces pratiques couvrent tout le domaine de la conduite de l'exploitation : de la production à la commercialisation. Nous avons retenu six pratiques qui nous paraissent avoir un potentiel discriminant entre exploitations par rapport à la question posée : trois agronomiques (intensification, autonomie et fertilisation agrobiologique), trois économiques (capitalisation, spécialisation et commercialisation) (tableau 2).

Certes, ces pratiques relèvent du fonctionnement de l'exploitation; toutefois, certaines d'entre elles, notamment celles économiques, ne sont pas sans lien avec les caractéristiques structurelles des exploitations. Ces six pratiques retenues constitueront les variables de l'analyse quantitative. La variable fertilisation agrobiologique étant composée de trois items, sa note globale est la somme des scores de ces items. Le tableau 2 précise les critères de mesure des différentes variables et la méthode de calcul des scores des items de la variable fertilisation agrobiologique. Nous avons recouru par la suite à la méthode de l'Analyse en composantes principales (ACP), en utilisant le logiciel Modalisa, pour élaborer une typologie des logiques de fonctionnement des exploitations.

En ce qui concerne la durabilité, nous avons élaboré une grille d'indicateurs en tenant compte des spécificités de l'AB (Gafsi et Favreau, 2012). L'objectif fixé est de pouvoir comparer les exploitations en $\mathrm{AB}$ entre elles, et non avec les exploitations conventionnelles. Pour cela nous avons pris en compte les grands principes de l'AB selon l'approche globale présentée plus haut (au-delà du non-usage des intrants de synthèse, importance de la fertilisation agrobiologique, diversité et complémentarité entre les activités de culture et d'élevage, valorisation des ressources locales et ajustement aux conditions agro-écologiques locales), ainsi que quelques méthodes d'évaluation de la durabilité des exploitations agricoles, connues en France (Idea, Rad et Dialecte) ${ }^{3}$. La méthode d'évaluation élaborée et utilisée comporte trois dimensions (tableau 3) : agro-écologique, économique et socio-territoriale. Chaque dimension compte quatre composantes et chaque composante est renseignée par des indicateurs notés. La somme des notes des différents indicateurs constitue la note de la composante, et la somme des notes des composantes constitue la note de la dimension. Chaque dimension est notée sur cent points. Les poids des composantes au sein de chaque dimension sont à peu près équilibrés. Les deux premières composantes de la dimension agro-écologique concernent la gestion de la fertilité, qui est une thématique très importante dans l'AB ; les deux autres concernent la biodiversité et la gestion des ressources. Les deux premières composantes de la dimension socio-territoriale apprécient le côté interne à l'exploitation de la dimension sociale; les deux autres portent sur le côté externe. Les deux aspects ont un poids équivalent. Enfin, la viabilité occupe une place importante dans la dimension économique; elle a une note plus élevée que les autres composantes.

Suivant le même principe que celui d'IDEA (Vilain, 2008), la note globale de la durabilité d'une exploitation est celle de la dimension limitante (la plus petite note des trois dimensions). Ce principe permet d'avoir la simultanéité des trois dimensions et donc une approche intégrée de la durabilité. Il évite aussi le problème de substituabilité des dimensions, qui relève d'une durabilité faible (Godard, 1994). Les scores obtenus pour chaque composante sont importants à analyser pour identifier les facteurs affectant la durabilité de l'exploitation dans la dimension concernée.

3. IDEA : Indicateurs de durabilité des exploitations agricoles (Vilain, 2008) ; RAD : Réseau agriculture durable, en Bretagne (RAD, 2001) ; est élaborée par le Réseau d'agriculture durable ; DIALECTE : Diagnostic agro-environnemental des CTE (http://dialecte.solagro.org/qui.php, consulté en octobre 2013). 
RECHERCHES

Mohamed GAFSI, Jean-Luc FAVREAU

Tableau 3. Grille d'évaluation de la durabilité des exploitations agricoles en $A B$

\begin{tabular}{|c|c|c|c|}
\hline & Composante & Indicateurs & Score \\
\hline \multirow{22}{*}{ 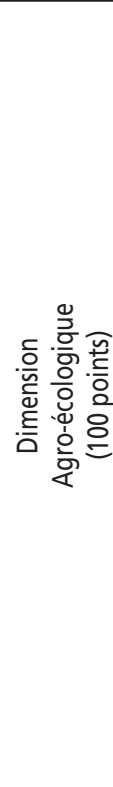 } & \multirow{6}{*}{$\begin{array}{l}\text { Gestion de la fertilité } \\
\text { du sol (19) }\end{array}$} & 1. Pression d'azote apportée & 1 à 4 \\
\hline & & 2. Surface recevant plus de $100 \mathrm{~kg}$ d'azote/ha & -2 à 0 \\
\hline & & 3. Pression phosphorique & 0 à 4 \\
\hline & & 4. Surface recevant plus de $40 \mathrm{~kg}$ de phosphore/ha & -2 à 0 \\
\hline & & 5. Apports de fumier, composte et engrais verts & 0 à 8 \\
\hline & & 6. Restitution des pailles & 0 à 3 \\
\hline & \multirow{3}{*}{ Rotation (26) } & 7. Durée de rotation & 0 à 12 \\
\hline & & 8. Diversité des cultures dans l'assolement & -4 à 0 \\
\hline & & 9. Part de la culture principale de l'assolement & 0 à 8 \\
\hline & \multirow{6}{*}{ Biodiversité (25) } & 10.Pourcentage de légumineuses & 0 à 6 \\
\hline & & 11. Linéaire des haies et bandes enherbées permanentes & 1 à 5 \\
\hline & & 12. $\%$ de SAU en prairies permanentes et temporaires $>5$ ans & 0 à 6 \\
\hline & & 13. Diversité animale & 0 à 9 \\
\hline & & 14. Animaux : races locales ou adaptées aux conditions locales & 0 à 3 \\
\hline & & 15. Cultures: variétés locales et anciennes & 0 à 2 \\
\hline & \multirow{7}{*}{$\begin{array}{l}\text { Gestion des ressources } \\
\text { (30) }\end{array}$} & 16. Couverture du sol en hiver (en $\%$ de la SAU) & 0 à 6 \\
\hline & & 17. Part de la surface assolée travaillée sans labour & 0 à 6 \\
\hline & & 18. Part de la surface assolée soumise à érosion & 0 à 5 \\
\hline & & 19. Irrigation & 0 à 6 \\
\hline & & 20. Irrigation sur moins de $30 \%$ SAU & 0 à 1 \\
\hline & & 21. Consommation énergétique en équivalant fioul/ha & 0 à 5 \\
\hline & & 22. Valorisation d'énergies renouvelables sur la ferme & 0 à 2 \\
\hline \multirow{7}{*}{ 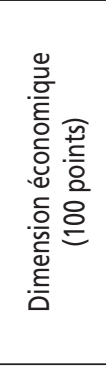 } & \multirow{3}{*}{ Viabilité (30) } & 23. Revenu agricole net /UTH & 0 à 20 \\
\hline & & $\begin{array}{l}\text { 24. Spécialisation : Produit de la production principale / Produit } \\
\text { brut global }\end{array}$ & 0 à 8 \\
\hline & & 25. Part du plus gros client & 0 à 2 \\
\hline & \multirow{2}{*}{ Autonomie (25) } & 26. Autonomie financière : annuités / EBE & 0 à 15 \\
\hline & & 27. Sensibilité aux aides : primes / EBE & 0 à 10 \\
\hline & $\begin{array}{l}\text { Transmissibilité } \\
\text { économique (20) }\end{array}$ & 28. Capital d'exploitation / UTH non salarié & 0 à 20 \\
\hline & $\begin{array}{l}\text { Efficience du processus } \\
\text { productif }(25)\end{array}$ & 29. (Production - intrants) / production & 0 à 25 \\
\hline \multirow{14}{*}{ 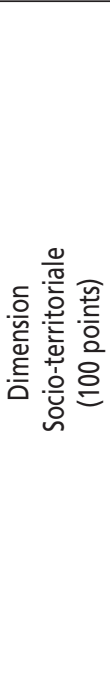 } & \multirow{4}{*}{$\begin{array}{l}\text { Conditions du travail } \\
\text { et développement } \\
\text { humain (26) }\end{array}$} & 30. Nombre de jours de congé / an & 0 à 8 \\
\hline & & 31. Nombre de semaines surchargées / an & 0 à 7 \\
\hline & & 32. Nombre de jours de formation / an & 0 à 6 \\
\hline & & 33. Ferme pédagogique, accueil de groupes, stages & 0 à 5 \\
\hline & \multirow{4}{*}{$\begin{array}{l}\text { Qualité de vie } \\
\text { et métier (24) }\end{array}$} & 34. Satisfaction du niveau de vie (revenu et avantages) & 0 à 10 \\
\hline & & 35. Satisfaction de la qualité de vie et du métier & 0 à 6 \\
\hline & & 36. Sentiment de ne pas être isolé & 0 à 3 \\
\hline & & 37. Perspective de transmission de l'exploitation & 0 à 5 \\
\hline & \multirow{5}{*}{$\begin{array}{l}\text { Contribution } \\
\text { à l'économie locale (26) }\end{array}$} & 38. Création d'emplois sur les 5 dernières années & 0 à 3 \\
\hline & & 39. Nombre d'emplois salariés (permanent ou temporaire) & \\
\hline & & 40. Surface par UTH / référence du Plan Agricole Départemental & \\
\hline & & 41. \% du CA commercialisé en circuits courts & 0 à 8 \\
\hline & & $\begin{array}{l}\text { 42. Agrotourisme } \\
\text { 43. Valorisation du patrimoine bâti, des paysages, sentiers }\end{array}$ & 0 à 3 \\
\hline & Implication sociale (24) & $\begin{array}{l}\text { 44. Implication et responsabilité dans des organisations locales } \\
\text { 45. Organisation, travail et investissements collectifs } \\
\text { 46. Implication dans les réseaux professionnels (CIVAM, Groupe- } \\
\text { ment d'agriculteurs biologiques, réseau d'expérimentation, etc.) }\end{array}$ & $\begin{array}{l}0 \text { à } 8 \\
0 \text { à } 8 \\
0 \text { à } 8\end{array}$ \\
\hline
\end{tabular}

Source : les auteurs 


\section{Résultats}

\section{Typologie des logiques de fonctionnement}

Les résultats de l'ACP montrent que cinq des six variables structurent les deux premiers axes. Les variables « capitalisation» et « commercialisation » ont le plus contribué au premier axe ; ce qui fait que cet axe sera plutôt qualifié d'axe économique. Le deuxième axe est structuré par les variables « intensification », « autonomie » et en partie «spécialisation»; il s'agit donc d'un axe plutôt agronomique (figure 1).

Trois groupes d'exploitations se dégagent de l'ACP, toutes productions confondues, correspondant à trois grandes logiques de fonctionnement. La première est qualifiée « d'entrepreneuriale » $(\mathrm{A})$, la deuxième de « recherche d'équilibre » $(\mathrm{B})$ et la troisième de « modernisation » $(\mathrm{C})$. Le tableau 4 présente le nombre d'exploitations concernées par chaque logique et leurs principales caractéristiques.
Figure 1. Typologie des exploitations selon les résultats de l'ACP

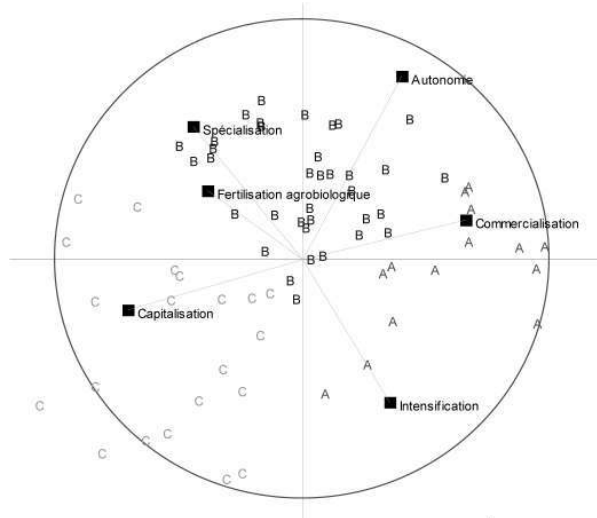

Note : premier plan constitué des facteurs 1 - horizontal - et 2 - vertical

Source : les auteurs.

Tableau 4. Caractéristiques des exploitations selon leurs logiques de fonctionnement

\begin{tabular}{lcccc}
\hline & $\begin{array}{c}\text { Logique } \\
\text { entrepreneuriale }\end{array}$ & $\begin{array}{c}\text { Logique de } \\
\text { recherche } \\
\text { d'équilibre }\end{array}$ & $\begin{array}{c}\text { Logique de } \\
\text { modernisation }\end{array}$ & Test F \\
\hline Nombre d'exploitation & 17 & 36 & 21 & \\
SAU moyenne (ha) & 25 & 87 & 126 & $13,92 *$ \\
Âge moyen de chef d'exploitation & 48 & 50 & 46 & 1,71 \\
UTH moyen & 1,6 & 1,6 & 1,7 & 0,34 \\
CA moyen (en milliers d' $€$ ) & 57 & 84 & 159 & $19,07^{*}$ \\
EBE moyen (en milliers d'€) & 24 & 34 & 60 & $11,15^{*}$ \\
Installation en hors cadre familial (\% par type) & 64 & 36 & 9 & \\
& & & & \\
Intensification en azote (kg d'azote/ha) & 98 & 62 & 75 & $10,21^{*}$ \\
$\begin{array}{l}\text { Autonomie (\% intrants/CA global) } \\
\text { Fertilisation agrobiologique (note sur 29) }\end{array}$ & 29 & 23 & 49 & $24,07^{*}$ \\
$\begin{array}{l}\text { Capitalisation : capital moyen d'exploitation / UTH } \\
\text { (milliers d' } € \text { ) }\end{array}$ & 18 & 25 & 23 & $8,23^{*}$ \\
$\begin{array}{l}\text { Spécialisation } \\
\text { (\% production principale/ CA global) }\end{array}$ & 48 & 96 & 140 & $29,24^{*}$ \\
$\begin{array}{l}\text { Commercialisation } \\
\text { (\% vente en circuits courts / CA global) }\end{array}$ & 74 & 83 & 74 & $12,56^{*}$ \\
\hline
\end{tabular}

Note: ${ }^{*} p<0,001$ 
La logique entrepreneuriale (A) est celle poursuivie, le plus souvent, par les exploitations de petite SAU, que ce soit en maraîchage (les 8 exploitations), en grandes cultures (4 exploitations) ou en caprin (3 exploitations). Installés souvent en hors cadre familial, les agriculteurs disposent de peu de moyens pour pouvoir engager de gros investissements et de modernisation de l'outil de production. Ils poursuivent une stratégie de création de la valeur ajoutée pour dégager un revenu acceptable à partir de petites structures. Pour y arriver, les trois quarts d'entre eux développent la transformation et la vente en circuits courts. Certains agriculteurs cherchent à aller plus loin dans les performances techniques, en améliorant les rendements, ce qui les amène à intensifier fortement leurs pratiques (plus de 150 unités d'azote/ha, en maraîchage).

La logique de recherche d'équilibre (B) est celle médiane, entre les deux premières (entrepreneuriale et de modernisation), sur l'axe 1 du plan de l'ACP (figure 1). Mais elle est plus portée par l'axe 2, notamment vers le pôle de recherche d'autonomie et de fertilisation agrobiologique. Elle concerne de manière à peu près équitable 5 principales orientations technico-économiques : bovins lait (6) et viande (8), ovin viande (7) et caprin (5), grandes cultures (9). Les dimensions en termes de SAU des exploitations sont globalement moyennes. Les agriculteurs s'inscrivent dans une démarche de recherche d'équilibre et de compromis entre plusieurs objectifs de différentes natures (économique, agronomique, sociale, identitaire, etc.). Ils affichent des objectifs de rentabilité, mais ceci ne conduit pas, pour autant, à une logique de court terme. Leurs pratiques agronomiques se caractérisent par une intensification modérée, une recherche d'autonomie en intrants, une pression modérée sur les ressources et une maîtrise des investissements. Certains agriculteurs essaient d'aller le plus possible vers des pratiques agronomiques relevant d'une intensification écologique (légumineuses dans des rotations de céréales, cultures associées, agroforesterie, etc.). Dans le groupe d'exploitations poursuivant cette logique, on peut distinguer les exploitations de grandes cultures qui pratiquent souvent la commercialisation en circuit long (80\% des exploitations), de celles d'élevage dont les productions sont valorisées principalement en circuits courts (environ $60 \%$ du chiffre d'affaires).

La logique de modernisation (C) est adoptée souvent par des exploitations de grande dimension, en termes de SAU et de chiffre d'affaires, vendant toutes ou quasiment toutes leurs productions en circuits longs. Les orientations technico-économiques dominantes sont les grandes cultures (10 exploitations) et l'élevage ovin lait (6 exploitations). Les 5 autres exploitations sont réparties comme suit : 2 en bovin viande, 2 granivores et 1 en bovin lait. Les agriculteurs poursuivent une politique de modernisation de l'outil de production et de renouvellement rapidement, ce qui se traduit par l'augmentation du niveau de capitalisation (capital d'exploitation par UTH). La plupart d'entre eux mettent en avant des objectifs élevés de productivité et de revenu. Ils n'hésitent pas à recourir à d'importants achats d'intrants (engrais et d'aliment pour le bétail), rendant ainsi leurs exploitations beaucoup moins autonomes. Quelques agriculteurs ont des pratiques intensives (fertilisation allant jusqu'à 140 unités d'azote/ha sur du blé, trois agnelages en deux ans, rotations de 3-4 ans avec les cultures de blé et soja).

\section{Logiques de fonctionnement et durabilité des exploitations}

Globalement, les résultats montrent que les exploitations biologiques de notre échantillon ont une durabilité plutôt moyenne (54/100). La dimension limitante est souvent la dimension de la durabilité 
socio-territoriale. Cette appréciation générale de la durabilité cache une grande diversité entre exploitations selon leurs orientations technico-économiques ou leurs logiques de fonctionnement. Les exploitations d'élevage sont plus durables agro-écologiquement que celles des grandes cultures (75 contre 54/100). Les dernières sont par contre plus durables au niveau économique (61 contre 53/100). Les scores de la durabilité socio-territoriale sont les mêmes dans les deux grands types d'exploitations. Les exploitations d'élevage ont de moins bonnes conditions de travail (moins de temps libre, davantage de semaines surchargées), mais elles ont une meilleure contribution à l'économie locale (davantage de commercialisation en circuits courts).

$\mathrm{Si}$ on se réfère aux logiques de fonctionnement, les différences de scores de durabilité sont significatives dans les deux dimensions : agro-écologique et économique. Elles ne le sont pas par contre concernant la durabilité socio-territoriale (tableau 5). La durabilité agro-écologique est plus élevée chez les exploitations qui ont une logique de recherche d'équilibre que chez celles qui suivent les deux autres logiques. Nous l'avons vu sur le plan de l'ACP, elles sont situées à l'opposé de la pratique d'intensification, et plutôt proche des pratiques d'autonomie et de fertilité agrobiologique. Soulignons toutefois que les scores des deux autres logiques ne sont pas non plus trop faibles; ce qui confirme l'appréciation générale de la durabilité agro-écologique de l'AB. La durabilité économique est plus élevée chez les exploitations de la logique entrepreuneuriale, mais elle est plus faible chez les exploitations de la logique de modernisation. Ce résultat est quelque peu paradoxal ; on s'attendait à l'inverse : une durabilité économique plus forte chez les agriculteurs modernistes que chez les entrepreneurs installés sur de petites structures. Mais ceci s'explique notamment par la note de transmissibilité de l'exploitation qui favorise ces derniers et pénalise globalement les exploitations de la logique de modernisation. Un autre fait saillant est le bon score de durabilité sur les trois dimensions des exploitations qui suivent la logique de recherche d'équilibre. Certes, elles ont une durabilité économique moins élevée que les exploitations de la logique entrepreneuriale ; mais elles ont en contrepartie des scores élevés au niveau des deux autres dimensions. Si l'on se base sur le principe de l'appréciation de la durabilité par le score de la dimension limitante, la logique de recherche d'équilibre serait la plus durable avec un score de 59,5 contre 55,19 et 54 respectivement pour les logiques de modernisation et entrepreneuriale.

Il est nécessaire, cependant, d'entrer dans le détail des composantes de la

Tableau 5. Moyennes des notes des dimensions de durabilité des trois logiques de fonctionnement

\begin{tabular}{lccc}
\hline & $\begin{array}{c}\text { Dimension } \\
\text { agro-écologique* }\end{array}$ & $\begin{array}{c}\text { Dimension } \\
\text { économique** }\end{array}$ & $\begin{array}{c}\text { Dimension } \\
\text { socio-territoriale }\end{array}$ \\
\hline Logique entrepreneuriale & 62,65 & 69,53 & 54,00 \\
Logique de recherche d'équilibre & 73,81 & 59,75 & 59,50 \\
Logique de modernisation & 62,95 & 56,33 & 55,19 \\
Total échantillon & 68,16 & 61,03 & 57,01 \\
& & & \\
Test F & $8,33^{* *}$ & $3,25 *$ & 1,5 \\
\hline
\end{tabular}

Note: ${ }^{*} p<0,05,{ }^{* *} p<0,001$ 
durabilité pour mieux comprendre les raisons des différences et des similitudes entre les différentes logiques de fonctionnement.

Les exploitations engagées dans la logique de recherche d'équilibre ont une durabilité plus élevée, avec les scores les plus hauts dans 6 composantes sur 12 (figure 2). Elles ont des notes supérieures dans les quatre composantes agro-écologiques et dans deux composantes socio-territoriales : conditions de travail et qualité de vie. Le bon niveau d'équipement, et probablement une certaine organisation du travail expliquent l'excellente note de condition de travail, malgré la pratique de la transformation et la vente en circuits courts. Ces exploitations ont par contre le score de viabilité le plus faible malgré la commercialisation en circuits courts pour beaucoup d'exploitations d'élevage. Bien que les agriculteurs ne suivent pas une politique de modernisation assez affirmée, comparés aux agriculteurs modernistes, le poids des annuités d'emprunts pénalise la viabilité. Grâce à des structures de taille suffisante et une capitalisation mesurée, les exploitations ont une bonne note de transmissibilité.

Les exploitations engagées dans la logique entrepreneuriale ont des résultats similaires en matière de viabilité économique. L'effet de la vente en circuits courts semble être important pour la viabilité, en permettant aux agriculteurs de capter la valeur ajoutée de la transformation (s'il $\mathrm{y}$ en a) et de la commercialisation. Mais cette importance n'est pas aussi suffisante pour que la viabilité de ces exploitations dépasse celle des exploitations de la logique de modernisation. La vente en circuits courts permet, par ailleurs, d'améliorer la note de la contribution à l'économie locale. Par contre, les exploitations poursuivant la logique entrepreneuriale ont les scores les plus élevés pour les trois autres composantes de la durabilité économique. Elles se distinguent surtout par la transmissibilité et l'autonomie financière, reflétant à la fois leur petite taille, une capitalisation faible, et une modernisation lente de l'outil de production limitant le recours

Figure 2. Notes des composantes de durabilité pour chaque type de logiques de fonctionnement

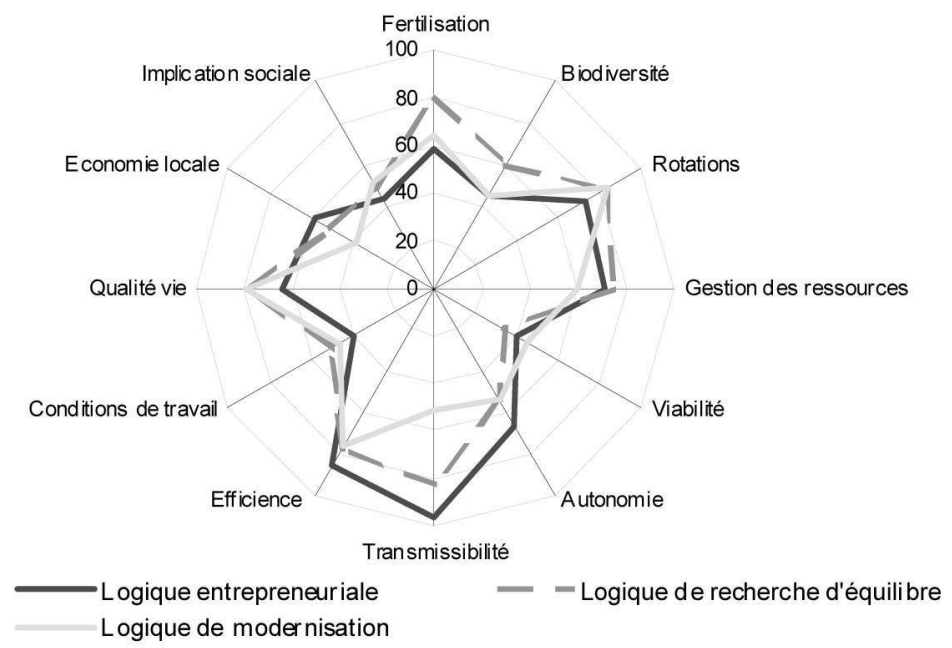

Note : notes relatives exprimées en \% par rapport à la note maximale de l'échelle 
à l'emprunt. La note élevée de la composante efficience est en lien avec la stratégie de création de la valeur ajoutée en pratiquant de la vente en circuits courts. L'effet d'intensification forte dans certaines exploitations s'est fait sentir au niveau des scores des composantes agro-écologiques, notamment la fertilisation et les rotations.

Les exploitations engagées dans la logique de modernisation se caractérisent par le score le plus élevé en viabilité économique, bien qu'il reste de niveau moyen. Mais les scores de la transmissibilité sont mauvais. Ceci est expliqué par la grande taille des exploitations en terme de SAU et une politique de mécanisation et de modernisation assez poussée nécessitant un recours systématique à l'emprunt. Excepté la biodiversité, les scores de durabilité agro-écologique sont plutôt assez bons. C'est pourtant dans ce groupe que l'on trouve les exploitations qui correspondent le plus à la tendance « conventionnelle » de l'AB, mais la présence des exploitations d'élevage, certes de grande SAU, mais moins intensives, équilibre les scores. La faible participation à l'économie locale est expliquée, d'une part, par la vente en circuits longs (à $84 \%$ du chiffre d'affaires), et d'autre part, par le manque de contribution à l'emploi.

\section{Discussion et conclusion}

L'AB est caractérisée depuis longtemps par une diversité de réseaux et de conceptions (Pernin, 1998 ; Leroux, 2011). Le récent de développement du secteur a conforté cette hétérogénéité. Il est important de saisir cette diversité pour traiter les questions comme la durabilité, afin de dépasser les appréciations générales et englobantes qui cachent les nuances et les différences dans un secteur hétérogène.

Le premier objectif de cette étude était de montrer, en mobilisant la notion de logique de fonctionnement, la pluralité de modèles et de conceptions de l'AB au niveau des exploitations agricoles. Les résultats confirment bien cette diversité de modèles et mettent en avant trois traits saillants : premièrement, cette diversité tranche bien avec la thèse de la bifurcation de l'AB en deux : une «éthique » et une autre « économique » (Guthman, 2004 ; Guptill, 2009 ; Lockie et Halpin, 2005). Elle permet de dépasser ainsi l'impasse de stéréotype qui caractérise le raisonnement de cette dichotomie (Streith et al., 2011). Elle montre au contraire une pluralité beaucoup plus importante de modèles. Déjà l'AB dite « éthique » est bien hétérogène et les nouveaux arrivants soupçonnés de pratiquer une bio « conventionnelle» sont aussi très hétérogènes et n'adhèrent pas forcément moins aux objectifs et principes de l'AB que les plus anciens agriculteurs (Padel, 2008).

Deuxièmement, la typologie de logiques de fonctionnement des exploitations étudiées présente une certaine proximité avec des typologies identifiées dans la littérature. Ainsi, deux types de logiques de fonctionnement correspondent, à quelques différences près, à deux des trois types identifiés par Darnhofer et al. (2010a). La logique médiane de recherche d'équilibre ressemble à celle qualifiée par les auteurs de «professionnalisation »; et celle de modernisation est proche de celle de « conventionalisation ». Sauf que le degré de conventionalisation est à nuancer pour les exploitations de la logique de modernisation. En effet, bien que leurs critères de dimension et de capitalisation soient les plus élevés dans l'échantillon, ceux-ci restent tout de même moins élevés que ceux des grandes exploitations conventionnelles (Agreste, 2012). La même remarque est valable aussi pour les critères de pratiques agronomiques, en prenant en compte tout de même que certaines exploitations présentent des pratiques de conventionalisation : simplification des assolements et rotations (rotations courtes, exemple : soja, soja, blé, sur parties irriguées), une 
pression parfois importante sur les ressources (irrigation, problèmes d'érosion). La logique entrepreneuriale n'a aucune proximité avec la troisième logique, dite « artisanale », de Darnhofer et al. (2010a). Elle est, par contre, en parfaite cohérence avec le type de "producteurs entrepreneurs » de Morel et Le Guen (2003).

Troisièmement, les résultats montrent l'effet indirect, mais important des variables structurelles (orientation et taille) dans certaines logiques de fonctionnement. Notre choix de départ d'étudier un ensemble d'exploitations aux orientations technico-économiques diverses nous semble pertinent. Il permet de mettre en évidence des logiques transversales à partir des variables se référant aux pratiques des agriculteurs. Les résultats ont montré effectivement que les logiques sont globalement transversales à plusieurs orientations de production ; mais des fois certaines logiques sont plus présentes dans une orientation plutôt que dans une autre (par exemple, la logique entrepreneuriale dans la production maraîchère). Cela révèle tout de même le poids du système de production dans l'orientation des logiques de fonctionnement. Une recherche plus approfondie concernant cette démarche typologique apporterait des résultats intéressants en opérant système de production par système de production, et en affinant pour chacun la méthode d'analyse ; ceci permettrait de révéler les différences de logiques au sein même des exploitations de même orientation de production.

Notons enfin, et en termes de perspective, que les résultats de cette étude ont été centrés sur une analyse synchronique des logiques de fonctionnement des exploitations. Mais ces logiques ne sont pas figées ; elles font l'objet de changement dans le cadre de la dynamique des agriculteurs et les processus d'innovation qu'ils engagent pour tenter de résoudre les problèmes techniques, économiques ou/et organisationnels qu'ils rencontrent dans la conduite de leurs exploitations. Une analyse diachronique permettrait de saisir les dynamiques de changement de pratiques agricoles. Ces dynamiques peuvent rester dans le cadre de la même logique, comme elles peuvent aussi «déborder »de ce cadre ; et dans ce cas-là, elles poussent à changer de logique de fonctionnement.

Le deuxième objectif de cette étude était de montrer que la durabilité varie en fonction des logiques de fonctionnement des exploitations. Notre tentative de caractériser la durabilité des exploitations en $\mathrm{AB}$ à partir de leurs logiques de fonctionnement semble porter ses fruits. En effet, les résultats ont montré que les différences de durabilité entre les trois logiques de fonctionnement sont significatives au niveau des dimensions de durabilité agro-écologique et économique. Ces résultats permettent de tirer deux principaux enseignements : (i) en matière de développement de l' $\mathrm{AB}$, le résultat important est l'existence bon nombre d'exploitations (logique de recherche d'équilibre, représentant presque la moitié de l'échantillon) alliant une structure de taille suffisante à des pratiques agronomiques visant l'autonomie et la valorisation des équilibres agrobiologiques, avec pour certains des efforts de modernisation et d'organisation, ainsi qu'une commercialisation en circuits courts les inscrivant dans un processus de développement territorial. Ces exploitations semblent donc réussir par différents arbitrages à concilier des objectifs divers et aboutir à une bonne performance globale (Reynaud, 2006). (ii) Le phénomène de conventionalisation, source de beaucoup d'inquiétudes pour des acteurs traditionnels de l'AB, se traduit dans les scores de durabilité de certaines exploitations de la logique de modernisation, de grande taille et commercialisant en circuits longs : problèmes de biodiversité, de gestion des ressources, de transmissibilité économique. Mais, contrairement à ce que l'on aurait pu 
attendre, les scores de certains indicateurs ne sont pas très différents de ceux d'autres logiques (rotations, efficience économique, implication sociale). On peut expliquer ces faibles écarts par le fait que l'agriculture de la Région Midi-Pyrénées, de par ses caractéristiques pédoclimatiques et sociales (exploitations familiales, etc.), ne se prête pas à une conventionalisation très poussée comme on aurait pu le voir dans des régions d'élevage (Bretagne) ou céréalières (Île-de-France). Une autre explication réside sans doute dans la question des échelles de notation et des pondérations dans notre méthode, qui ne sont probablement pas assez discriminantes. De nouveaux travaux permettront d'affiner encore plus cette méthode.

\section{RÉFÉRENCES BIBLIOGRAPHIQUES}

Agence Bio (2012). L'agriculture biologique en France. Chiffres clés 2011. Site www. agencebio.org, consulté en avril 2013.

Agreste (2012). L'agrandissement des exploitations se poursuit. Agreste Midi-Pyrénées Données, $\mathrm{n}^{\circ} 69$, p. 1-8.

Amblard H., Bernoux P., Herreros G., Livian Y.-F. (2005). Les nouvelles approches sociologiques des organisations. Paris, Seuil.

Bréchet J.-P., Schieb-Bienfait N. (2006). Projets et pouvoirs dans les régulations concurrentielles : la question de la morphogenèse d'une filière biologique. Revue d'économie industrielle, $\mathrm{n}^{\circ} 113$, p. 9-32.

Brossier J., Petit M. (1977). Pour une typologie des exploitations agricoles fondée sur les projets et les situations des agriculteurs. Économie rurale, $\mathrm{n}^{\circ} 122$, p. 31-40.

Brossier J., Chia E., Marshall E., Petit M. (1997). Gestion de l'exploitation agricole familiale. Éléments théoriques et méthodologiques. Cnerta, Dijon.

Capillon A. (1988). Jugement des pratiques et fonctionnement des exploitations. In Jollivet M., (dir.), Pour une agriculture diversifiée (p. 124-133), Paris, L'Harmattan.

Capillon A. (1993). Typologie des exploitations agricoles. Contribution à l'étude des problèmes techniques. Thèse de doctorat en sciences agronomiques, Paris, INAPG.

Darnhofer I., Lindenthal T., Bartel-Kratochvil R., Zollitsch W. (2010a). Conventionalisation of organic farming practices: from structural criteria towards an assessment based on organic principles. Agronomy for sustainable development, $\mathrm{n}^{\circ} 30$, p. 67-81.

Darnhofer I, Fairweather J., Moller H. (2010b). Assessing a farm's sustainability : insights from resilience thinking. International journal of agricultural sustainability, vol. 8, $\mathrm{n}^{\circ} 3$, p. 186-198.

Gafsi M., Favreau J.L. (2012). Indicator-based method for assessing organic farming sustainability. In Marta-Costa A. A., Silva E. (dir.), Methods and procedures for building sustainable farming systems. Aplications in the european context (p. 175-189), London, Springer.

Gafsi M., Le Tron S., Mouchet C. (2010). Organic farming is it a sustainable agriculture? Communication au Colloque international ISDA, Innovation and Sustainable Development in Agriculture and food, 28 au 30 juin 2010, Montpellier.

Girard N. (2006). Catégoriser les pratiques d'agriculteurs pour reformuler un problème en partenariat. Une proposition méthodologique. Cahiers Agricultures, vol. 15, $\mathrm{n}^{\circ} 3$, p. 261-272.

Godard O. (1994). Le développement durable : paysage intellectuel. Nature, Sciences, Sociétés, vol. 2, n 4, p. 309-324.

Godard O., Hubert B. (2002). Le développement durable et la recherche scientifique à l'INRA. Paris, INRA Édition.

Goldberger J. R. (2011). Conventionalisation, civic engagement, and the sustainability of organic agriculture. Journal of Rural Studies, ${ }^{\circ} 27$, p. 288-296. 
Guptill A. (2009). Exploring the conventionalisation of organic dairy : trends and counter-trends in upstate New York. Agriculture and Human Values, $\mathrm{n}^{\circ} 26$, p. 29-42.

Guthman J. (2004). The trouble with "organic live" in California: a rejoinder to the "conventionalisation" debate. Sociologia ruralis, $\mathrm{n}^{\circ} 44$, p. 301-316.

Hansen J. W., Jones J. W. (1996). A systems framework for characterizing farm sustainability, Agricultural Systems, $\mathrm{n}^{\circ}$ 51, p. 185-201.

Kratochvil R., Leitner H. (2005). The "trap of conventionalisation”: organic farming between vision and reality. XXI congress of ESRS, 22-27 août 2005, Keszthely, Hongrie.

Lamine C., Bellon S. (2009). Conversion to organic farming: a multidimensional research object at the crossroads of agricultural and social sciences. A review, in Agronomy for sustainable development, $\mathrm{n}^{\circ} 29$, p. 97-112.

Leroux B. (2011). L'agriculture biologique et l'alternative. Contribution à l'anthropologie politique d'un monde paysan en devenir. Thèse de Doctorat en Sociologie, EHESS, $408 \mathrm{p}$.

Lockie S., Halpin D. (2005). The "conventionalisation" thesis reconsidered: structural and ideological transformation of Australian organic farming. Sociologia ruralis, vol. 45, $\mathrm{n}^{\circ} 4$, p. 284-307.

Morel B., Le Guen R. (2003). Une typologie compréhensive pour analyser la dynamique des producteurs biologiques. Recherches pour et sur le développement régional, Actes du séminaire de Montpellier des 17 et 18 décembre 2002, INRA, p. 279-291.

Padel S. (2008). Value of organic producers converting at different times : results of a focus group study in five European countries. International Journal of Agricultural Resources, Governance and Ecology, vol. 7, $\mathrm{n}^{\circ} 1-2$, p. 63-77.

Pernin J.-L. (1998). Identités et mutations identitaires d'un méso-système économique : le cas de l'agriculture biologique en France. Économie appliquée, vol. 51, n 2 , p. 139-168.

Rad (2001). Évaluer la durabilité d'un système de production. Rennes, Cahier technique de l'agriculture durable, Réseau Agriculture Durable en Bretagne, 60 p.

Reynaud E. (2006) (dir.). Le développement durable au cour de l'entreprise. Paris, Dunod.

Rigby D., Caceres D. (2001). Organic farming and the sustainability of agriculture systems. Agricultural Systems, ${ }^{\circ}$ 68, p. 21-40.

Ruault C. (2006). Le conseil aux agriculteurs « bio » : un analyseur des interrogations et évolutions du conseil en agriculture. In Rémy J., Brives H., Lemery B. (dir.), Conseiller en agriculture (p. 183-200), Dijon, Educagri.

Schieb-Bienfait N., Sylvander B. (2004). Filière biologique et logiques d'action : éléments d'analyse des difficultés de structuration d'une filière régionale. $13^{\mathrm{e}}$ conférence de l'AIMS, Normandie, Vallée de Seine, 2-4 juin, $30 \mathrm{p}$.

Streith M., Van Dam D., Nizet J. (2011). L'agriculture biologique comme champ en tension. Une approche dialectique. Communication au Colloque SFER/RMT DevAB, Les transversalités de l'agriculture biologique, Strasbourg, 23-24 juin.

Sylvander B., Bellon S., Benoit M. (2006). Facing the organic reality: the diversity of development models and their consequences on research policies. Joint Organic Congress, Odense, Danemark, 30-31 mai.

Thompson P. B. (1997). The varieties of sustainability in livestock farming. Sorensen, éd. J.T., p. 5-15, In Livestock farming systems: more than food production. Wageningen Pers, Wageningen EAAP publications 89.

Tuomisto H. L., Hodge I. D., Riordan P., Macdonald D. W. (2012). Does organic farming reduce environmental impacts? A meta-analysis of European research. Journal of Environmental Management, $\mathrm{n}^{\circ} 112$, p. 309-320.

Van Dam D. (2005). Les agriculteurs biologiques : la réappropriation de l'identité de fermier, in Actes du colloque « Faire Campagne », vol. 1, p. 61-76, Rennes, 17-18 mars.

Vilain L. (2008). La méthode IDEA : indicateurs de durabilité des exploitations agricoles. Guide d'utilisation. Dijon, Educagri, $184 \mathrm{p}$. 